\title{
Não basta fazer ou fazer bem! É preciso saber fazer bem e com rigor!
}

\author{
Uma pequena digressão pelo mundo da termofísica ${ }^{1}$
}

\author{
CARLOS NIETO DE CASTRO
}

\begin{abstract}
Introdução
As propriedades termofísicas de fluidos desempenham um papel crucial no desenvolvimento da Ciência Molecular dos Fluidos, seja no campo da interpretação das forças intermoleculares, seja no da obtenção de informação experimental indispensável à compreensão da estrutura e dinâmica do estado líquido e gasoso denso. Presentes na grande maioria dos processos químicos e bioquímicos, bem como no processamento de materiais, têm um papel muito importante na produção, separação e concentração, optimização e controle de qualidade de matérias-primas, produtos intermediários e finais. Consequentemente, têm sido o objecto da aplicação das teorias moleculares, baseadas na Termodinâmica Estatística, de forma a poderem ser calculadas a partir dos primeiros princípios.
\end{abstract}

Dai a importância que têm e sempre tiveram no desenvolvimento da sociedade contemporânea e da ciência, nomeadamente na física, na química e, mais recentemente, na bioquímica.

Aproveitando a oportunidade de uma mais que justa homenagem ao trabalho desenvolvido pelo Professor Jorge Calado, na área da Termodinâmica Molecular, decidi escrever um pouco sobre as razões de três décadas de investigação em Termofísica, desde o já longínquo início de 1972, dedicado àqueles que futuramente continuarão o esforço de investigação nesta área do conhecimento.
Dos fluidos simples aos complexos, em gamas de temperatura e pressão variadas, com casos de sucesso fácil e outros de quase insucesso, mas sempre com a certeza de que a actividade em boa hora inspirada por Jorge Calado está viva e de boa saúde.

\section{Porquê a Termofísica?}

O desenvolvimento científico e tecnológico, especialmente quando processos de transferência de massa e de calor, com ou sem reaç̧ão química estão presentes, necessita de valores de propriedades de fluidos puros e de suas misturas que podem ser obtidos de várias maneiras:

- por medição experimental, directa ou indirecta

- através de cálculo teórico ou semiempírico - correlação, previsão ou estimativa

\section{- por simulação computacional}

É completamente impossível medir experimentalmente todas as propriedades termofísicas, em todas as condições do diagrama de fases de cada componente ou duma mistura multicomponente de composição arbitrária. Esta atitude ideal está limitada pelo tempo e pelo custo incomportável de tal abordagem. Sendo assim, torna-se necessário desenvolver mecanismos que permitam, a partir dum mínimo de dados experimentais, gerar os valores das propriedades necessárias.
O objectivo mais importante dum cientista seria o cálculo das propriedades a partir dos primeiros princípios, produzindo algoritmos poderosos para serem aplicados na indústria ou nos laboratórios de instalações piloto, com um grau de confiança elevado. Contudo, o estado actual da teoria molecular de gases e líquidos, incluindo a zona fluida [1-4] põe uma barreira inultrapassável à qualidade dos métodos de previsão, pois estamos normalmente bloqueados por equações da mecânica estatística intratáveis ou insolúveis. Se já é difícil obter equaçōes que liguem o mundo macroscópico, real e mensurável, ao microcosmos, torna-se muito mais difícil resolver essas equações para modelos realísticos de forças intermoleculares de par e multicêntricas, mesmo para os gases diluídos e moderadamente densos.

Temos assim de desenvolver um conjunto de métodos de cálculo das propriedades termofísicas, baseados em soluções aproximadas das equações fundamentais, em extensões heurísticas da teoria ou em relações meramente empíricas.

A medição experimental, duma forma rigorosa, das propriedades de equilíbrio e, nomeadamente das propriedades de transporte de fluidos não é uma tarefa fácil [5]. Por exemplo, no caso das propriedades de transporte, e por se tratarem de propriedades que caracterizam e quantificam fenómenos de não equilíbrio, a simples imposição de gradiente ao sistema a estudar modifica o seu estado de equilíbrio. A evolução para um 
novo estado de equilíbrio pode envolver o fenómeno que pretendemos estudar (a condução térmica, p.ex.) e outros fenómenos que o acompanham e difíceis de distinguir do primeiro fenómeno (a radiação e a convecção, p.ex.). Os fenómenos existentes no fluido em estudo têm assim que ser muito bem modelados matematicamente e decompostos fisicamente, para que possamos confiar em que os resultados obtidos por um dado método experimental traduzam na realidade os valores das grandezas físicas que desejamos conhecer.

A escolha dum determinado método de mediçã̃o depende de variadíssimos factores, de entre os quais podemos destacar a exactidão (ou rigor) a obter, o intervalo de estados termodinâmicos a estudar, o tipo de fluidos ou misturas fluidas a estudar e os recursos económicos.

Qualquer destes aspectos permite escoIher um método de elevado rigor, primário, restrito a um pequeno numero de fluidos padrão, importante para um laboratório com responsabilidades metrológicas, como os laboratórios nacionais de metrologia, ou um método de rotina de controlo de qualidade duma fábrica.

Os métodos de medição podem classificar-se em absolutos e relativos, sendo os primeiros aqueles em que existe uma equação de trabalho completa, que modela rigorosamente o processo físico em causa, estabelecendo uma relação ideal entre as variáveis medidas e a propriedade de transporte (modelo ideal), e um conjunto de correcções ao modelo ideal que traduzem os afastamentos entre o modelo ideal e a situaçāo experimental real. Os métodos de medição relativos săo aquelos em que uma ou mais constantes têm de ser determinadas a partir duma ou várias calibrações, com dados pré-conhecidos e com exactidão conhecida de outro(s) fluido(s). Os instrumentos sâo projectados criteriosamente, mas não existe uma teoria do método que suporte esse dimensionamento.

Para os métodos de medição relativos a incerteza dos instrumentos que os utilizam é 2 a 3 vezes maior do que a obtida com os instrumentos absolutos. Eles são a base das medições efectuadas pela grande maioria dos laboratórios acreditados na área da metrologia, bem como pelos laboratórios de ensaios.

No caso da densidade, viscosidade, condutibilidade térmica, difusibilidades mássicas, capacidades calorífica, permissividade dieléctrica e velocidades do som existem hoje em dia métodos absolutos e relativos de pequena incerteza para condições de pressão e temperatura semelhantes às ambientes. No entanto a sua extensão a temperaturas e pressões mais elevadas ou a novos compostos requer a sua adaptação ou o desenvolvimento de novos métodos, com um rigor compativel com a aplicação desejada. 0 problema complica-se muito mais quando estudamos sistemas heterogéneos ou que envolvam reacção química. Veja-se por exemplo o estudo dos fluidos supercríticos [6] ou dos halocarbonetos [7] ou da medida de difusibilidades mássicas [8]. Neste último caso o desenvolvimento das técnicas de espectroscopia de correlação fotónica permitiu a sua aplicaçāo à determinação de difusibilidades mássicas e térmicas em sistemas heterogéneos, poliméricos ou não, bem como ao estudo da dinâmica das moléculas em condições perto dos pontos críticos de mistura [9], sendo contudo ainda discutivel a exactidão dos resultados obtidos.

\section{1. ${ }^{a}$ Conclusão - Torna-se necessário desenvolver técnicas de medida de propriedades de equilibrio e de trans- porte para certas regiões do diagrama de fases com importância tecnológica e científica, com características abso- lutas, e que permitam obter um con- junto de dados padrão de referência de fluidos seleccionados. Estes dados pa- drão poderão ser utilizados para cali- brações de instrumentos relativos e para testar as teorias moleculares e a possibilidade da previsão ou estimativa de propriedades.}

Retomando o problema da previsão de propriedades, convém ainda salientar que a observação de comportamentos universais dos fluidos, tipo estados correspondentes, permite estender os intervalos de temperatura e pressão, por vezes inacessiveis do ponto de vista ex- perimental, para certos fluidos puros ou misturas.

O estado actual dos métodos da estimativa semiempírica ou empírica de propriedades, recomenda um estudo criterioso das teorias moleculares existentes, suas possibilidades de solução e a procura de analogias de comportamentos que permitam o cálculo das propriedades com uma confiança razoável [4].

Uma das ferramentas mais poderosas de cálculo rigoroso das propriedades termofísicas, envolvendo no entanto métodos matemáticos solúveis, é a teoria alargada dos estados correspondentes (ECST). Esta teoria combina uma teoria dos estados correspondentes de base molecular com uma equaçāo de estado empírica, mas muito rigorosa. 0 potencial do princípio dos estados correspondentes ${ }^{2}$ é a sua capacidade de prever as propriedades dos fluidos com uma informação mínima sobre o sistema em estudo, se existirem dados suficientes sobre outros sistemas molecularmente semelhantes. 0 rigor desta abordagem reside na equação de estado empírica do fluido de referência, utilizada na definição do estado termodinâmico do fluido em estudo. Um dos exemplos mais recentes é a previsão das propriedades termofísicas de refrigerantes e misturas de refrigerantes, bem como de sais fundidos [10-13]. A principal limitação do ECST é a ausência de equaçōes de estado rigorosas, para os fluidos de referência mais adequados a cada caso.

Dada a sua importância na engenharia química, convém referir-se o problema das correlações de propriedades, ou da sua variação com temperatura, pressão ou composição. As correlações, apenas convenientemente utilizáveis para interpolaçōes, podem ser empiricas, como por exemplo as desenvolvidas por ajuste polinomial multiparamétrico, ou terem uma base teórica, como as desenvolvidas para os gases reais, moderadamente densos. No caso da base teórica poderemos ainda extrapolar com alguma garantia. A grande maioria das correlações até agora desenvolvidas, incluindo as equações de estado de fluidos puros ou de misturas, têm uma 
Tabela 1 - Fluidos estudados

\begin{tabular}{|c|c|}
\hline Fluidos simples & Ar, $N_{2}, n-C_{1}$ to $C_{4}$ \\
\hline Hidrocarbonetos & lineares e ramificados, alifáticos e aromáticos (incluindo terpenos) \\
\hline Refrigerantes & Derivados clorados e fluorados do $C_{1}, C_{2}$ e $C_{3}$, puros e em misturas \\
\hline Alcoóis & saturados \\
\hline Água e misturas aquosas & Água, água deuterada, água + etanol, água+aminas \\
\hline Vapores metálicos & $\mathrm{Li}, \mathrm{Na}, \mathrm{K}, \mathrm{Rb}, \mathrm{Cs}$ \\
\hline Soluções aquosas de electrólitos & $\mathrm{NaCl}, \mathrm{KCl}, \mathrm{CsCl}$ \\
\hline Sais fundidos & $\mathrm{KNO}_{3}, \mathrm{NaNO}_{3}$ \\
\hline Metais fundidos & em curso \\
\hline Soluções de polímeros & em curso \\
\hline
\end{tabular}

Tabela 2 - Propriedades estudadas

$\begin{array}{ll}\text { Transporte } & \begin{array}{l}\text { condutibilidade térmica, viscosidade, difusibilidade mássica e coeficiente de } \\ \text { auto-difusão, difusibilidade térmica }\end{array} \\ \text { Termodinâmicas } & \begin{array}{l}\text { densidade, capacidade calorífica, equilíbrio líquido vapor, tensão superficial, } \\ \text { solubilidade }\end{array} \\ \text { Dieléctricas } & \text { índices de refracção, permissividade relativa (constante dieléctrica) }\end{array}$

base empírica, pelo que se torna importante contribuir para o desenvolvimento de correlações de propriedades ou entre propriedades com base teórica.

\section{Conclusão - É fundamental desen- volver métodos de previsão/estimativa e correlações de propriedades com uma base teórica credível.}

Um processo alternativo ao uso de equações de estado para descrever o comportamento termodinâmico de sistemas fluidos, consiste na utilização de propriedades convencionais da termodinâmica clássica tais como as propriedades molares parciais e as propriedades de excesso [14]. Os valores destas pro- priedades calculados a partir de dados experimentais e tratados por modelos moleculares, contendo alguns parâmetros ajustáveis, têm sido utilizados com êxito para correlacionar dados, obter parâmetros moleculares [15] e contribuir para o esclarecimento de certos fenómenos observados.

Apesar da importância dos métodos acima descritos, métodos puramente termodinâmicos, como estudos da variação das propriedades acima mencionadas com a temperatura, a pressão e a composição, são também importantes pelo facto de que esta variação nas propriedades pode ser detectada e estudada independentemente de qualquer re- ferência a modelos. Neste último caso, a detecção de fenómenos singulares, tais como variações bruscas de tipos de agregação molecular e de empacotamento [16] ou a rotação das moléculas no estado líquido [17] e a larga gama de propriedades que se pode conseguir ao fazer variar a composição das misturas e utilizar diferentes condições de temperatura e pressão, tem sido a grande motivação de estudos deste tipo. A solvatação e o efeito de adição de sais (salting-out) na solubilidade de não-electrólitos em água são alguns aspectos adicionais que nos permitem compreender melhor as interacções moleculares no estado líquido [18] 
3. ${ }^{\text {a }}$ Conclusão - É fundamental utilizar determinações experimentais de várias propriedades que permitam obter informações detalhadas e sustentadas da estrutura das misturas líquidas e da solubilidade das substâncias na fase líquida.

Last, but not the least, a simulação computacional. 0 desenvolvimento da capacidade de cálculo dos computadores, bem como da sua capacidade de processamento paralelo, permite hoje em dia efectuar cálculos bastante rigorosos da estrutura e propriedades dos fluidos, mesmo de fluidos complexos, evidenciando por exemplo a existência de rotações moleculares impedidas, pontes de hidrogénio, estrutura de agregados moleculares (clusters) e calculando momentos dipolares e polarizabilidades, como no caso dos compostos fluorados derivados do etano (os fluorocarbonetos) [19] e propriedades de transporte de sais fundidos como a viscosidade, a difusão e a condutibilidade térmica [20]. Combinações criteriosas de métodos ab-initio, do campo reaccional autoconsistente (self-consistent-reactionfield), teoria funcional da densidade, métodos de Monte-Carlo e Dinâmica Molecular, permitem actualmente obter resultados ontem inimagináveis sobre os comportamentos dos agregados moleculares e das suas propriedades macroscópicas.

Apresenta-se na Tabela 1 uma lista dos fluidos estudados e na Tabela 2 as propriedades estudadas.

\section{Conclusão Final - Desde 1972 abordá-} mos todos estes campos, gradualmente e com grande rigor. Teria sido possível fazê-lo sem umas bases fortes? Penso que não! Investigar e inovar em Termofísica não é fácil, mas recompensa facilmente. No meu entender e dos meus colaboradores valeu e vale a pena. Assim a educação pré-universitária e universitária corresponda, não afugentando os bons estudantes das ciências exactas, nomeadamente da Química-Física e da Termodinâmica Molecular.

\section{Referências}

[1] K. Lucas, "Applied Statistical Thermodynamics", Springer Ve-lag (1991)

[2] G. Maitland, M. Figby, B. Smith, W. A. Wakeham, "Intermolecular Forces", Oxford (1981)

[3] J. W. Whalen, "Molecular Thermodynamics - A Statistical Approach", John Wiley \& Sons (1991)

[4] J. Millat, J. H. Dymond, C. A. Nieto de Castro, eds., "The Transport Properties of Fluids - Their Correlation, Prediction and EStimation", Cambridge University Press, London, (1996)

[5] W. A. Wakeham, A. Nagashima, J. V. Sengers, Eds., "Measurement of Transport Properties of Fluids", IUPAC Volume III on Experimental Thermodynamics, Blackwell Scientific Publications, Oxford (1991)

[6] T. J. Bruno, J. E. Ely, "Supercritical Fluid Technology - Reviews in Modern Theory and Applications" CRC Press (1991)

[7] C. A. Nieto de Castro, "Os Halocarbonetos e o Ambiente", Boletim da Sociedade Portuguesa de Química, 48, 16 (1993).

[8] H. J. V. Tyrrell, K. R. Harris, "Diffusion in Liquids", Butterworths (1984)

[9] R. Pecora, Ed., "Dynamic Light Scattering. Application of Photon Correlation Spectroscopy', Plenum Press (1985)

[10] I. M. Marrucho, "Extended Corresponding States Theory: Application for Polar Compounds and Their Mixtures", Ph.D. Thesis, IST, Portugal (1996)

[11] Byung Ik Lee and Michael G. Kesler, "A Generalized Thermodynamic Correlation Based on the Three-Parameter Corresponding States", AlChE Journal, 21, 510-527 (1975)
[12] N. Galamba, C. A. Nieto de Castro, I. Marrucho, J. Ely, "A Corresponding States Aproach for the Calculation of the Transport Properties of Uni-Univalent Molten Salts", High Temp. - High Press., 33, 397 (2001)

[13] N. Galamba, C. A. Nieto de Castro, I. Marrucho and J.F. Ely, 'A Corresponding States Approach for the Prediction of Surface Tension of Molten Alkali Halides", Fluid Phase Equilibria, 183-184, 239 (2001)

[14] M. M. Abbott and K. K. Nass, "Equations of State and Classical Solution Thermodynamics. Survey of the Connections", ACS Symp. Ser., 300, 1-40 (1986)

[15] J. M. Prausnitz, R. N. Lichtentbater and E. G. de Azevedo, "Molecular Thermodynamics of Fluid-Phase Equilibria", Prentice-Hall Inc. (1986)

[16] G. Doubéret and M. I. Davis, "Measurements, Analysis and Utility of Excess Molar(dV/dP)s", Chem. Soc. Rev., 22, 43 (1993)

[17] M. T. Barāo, C. A. Nieto de Castro, U. V. Mardolcar, "The Dielectric Constant of Liquid HFC 134a and HCFC 142b", Int. J. Thermophys., 17, 573 (1996)

[18] I. M. S. Lampreia and J. M. S. T. Neves, "UItrasonic Velocities and Isentropic Compressions of Triethylamine in Water and in Aqueous Tetraethylammonium Chloride Solutions from 15 to $35^{\circ} \mathrm{C}$, Termochimica Acta, 298, 65-74, (1997)

[19] B. J. Costa Cabral, R. C. Guedes, R. S. Pai-Panandiker, C. A. Nieto de Castro, "Hydrogen Bonding and Internal Rotation of Hydrofluorocarbons by Density Functional Theory', Phys, Chem. Chem. Phys., 3, 4200 (2001)

[20] N. Galamba, J. Ely, C. A. Nieto de Castro, "Transport Properties of Molten Sodium Chloride through Equilibrium Molecular Dynamics", (2003), to be submitted 